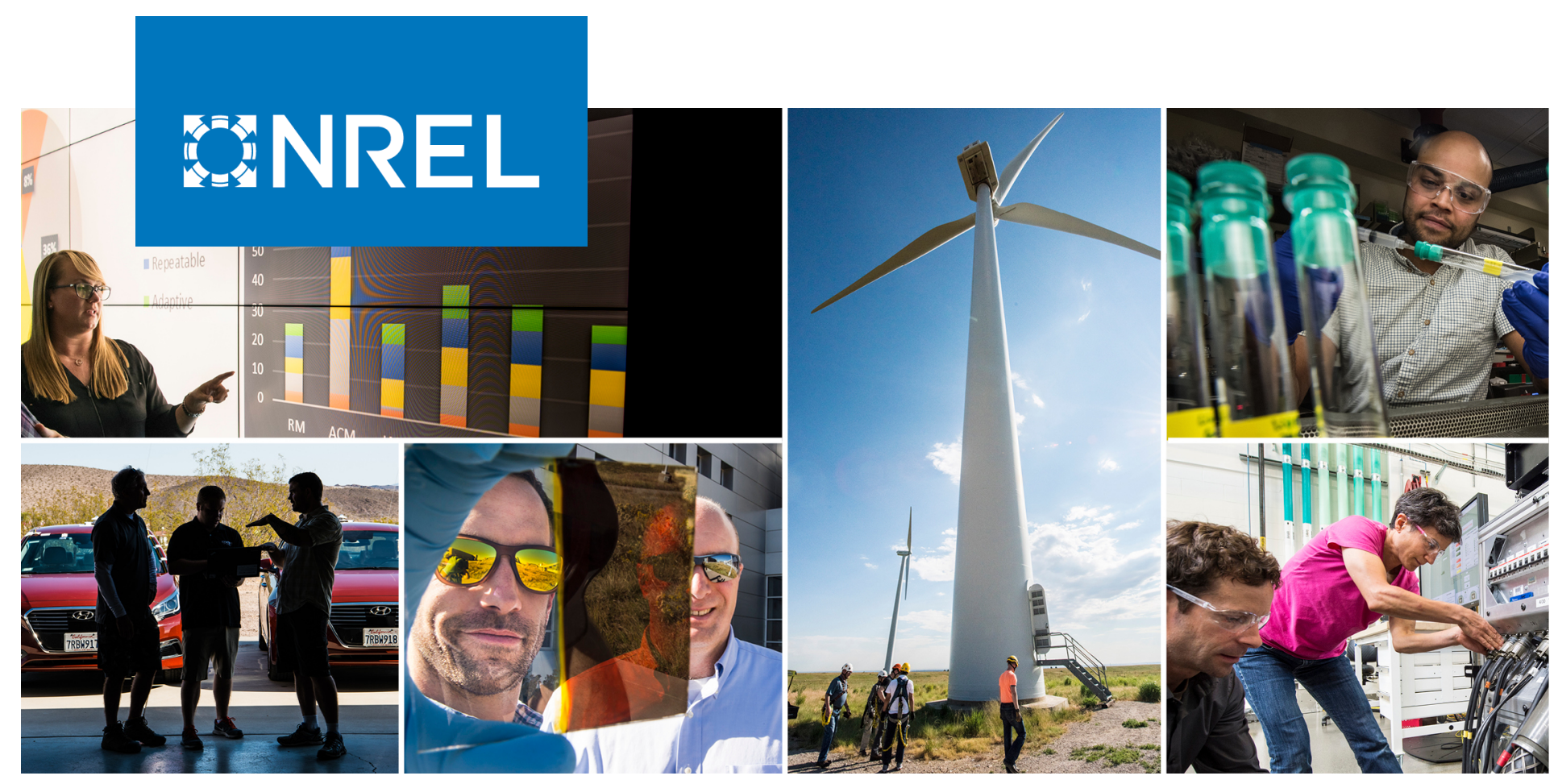

\title{
bifiPV2020 Bifacial Workshop: A Technology Overview
}

Elias Urrejola, ${ }^{1}$ Felipe Valencia, ${ }^{1}$ Edward Fuentealba, ${ }^{2}$ Chris Deline, ${ }^{3}$ Silvana Ayala Pelaez, ${ }^{3}$ Jenya Meydbray, ${ }^{4}$ Tori Clifford, ${ }^{4}$ Radovan Kopecek, ${ }^{5}$ and Joshua S. Stein ${ }^{6}$

1 ATAMOSTEC

2 University of Antofagasta

3 National Renewable Energy Laboratory

4 PV Evolution Labs

5 ISC-Konstanz

6 Sandia National Laboratories
NREL is a national laboratory of the U.S. Department of Energy Office of Energy Efficiency \& Renewable Energy

Operated by the Alliance for Sustainable Energy, LLC

This report is available at no cost from the National Renewable Energy Laboratory (NREL) at www.nrel.gov/publications.
Technical Report

NREL/TP-5K00-77817

October 2020 


\title{
GNREL
}

\section{bifiPV2020 Bifacial Workshop: A Technology Overview}

\author{
Elias Urrejola, ${ }^{1}$ Felipe Valencia, ${ }^{1}$ Edward Fuentealba, ${ }^{2}$ \\ Chris Deline, ${ }^{3}$ Silvana Ayala Pelaez, ${ }^{3}$ Jenya Meydbray, ${ }^{4}$ \\ Tori Clifford, ${ }^{4}$ Radovan Kopecek, ${ }^{5}$ and Joshua S. Stein ${ }^{6}$
}

1 ATAMOSTEC

2 University of Antofagasta

3 National Renewable Energy Laboratory

4 PV Evolution Labs

5 ISC-Konstanz

6 Sandia National Laboratories

\section{Suggested Citation}

Urrejola, Elias, Felipe Valencia, Edward Fuentealba, Chris Deline, Silvana Ayala Pelaez, Jenya Meydbray, Tori Clifford, Radovan Kopecek, and Joshua S. Stein. bifiPV2020 Bifacial Workshop: A Technology Overview. Golden, CO: National Renewable Energy Laboratory. NREL/TP-5K00-77817. https://www.nrel.gov/docs/fy21osti/77817.pdf.

NREL is a national laboratory of the U.S. Department of Energy Office of Energy Efficiency \& Renewable Energy Operated by the Alliance for Sustainable Energy, LLC

This report is available at no cost from the National Renewable Energy Laboratory (NREL) at www.nrel.gov/publications.

Contract No. DE-AC36-08GO28308
Technical Report NREL/TP-5K00-77817 October 2020

National Renewable Energy Laboratory 15013 Denver West Parkway Golden, CO 80401

303-275-3000 • www.nrel.gov 


\section{NOTICE}

This work was authored [in part] by the National Renewable Energy Laboratory, operated by Alliance for Sustainable Energy, LLC, for the U.S. Department of Energy (DOE) under Contract No. DE-AC3608GO28308. Funding provided by U.S. Department of Energy Office of Energy Efficiency and Renewable Energy Solar Energy Technologies Office. The views expressed herein do not necessarily represent the views of the DOE or the U.S. Government.

This report is available at no cost from the National

Renewable Energy Laboratory (NREL) at

www.nrel.gov/publications.

U.S. Department of Energy (DOE) reports produced

after 1991 and a growing number of pre-1991

documents are available

free via www.OSTI.gov.

Cover Photos by Dennis Schroeder: (clockwise, left to right) NREL 51934, NREL 45897, NREL 42160, NREL 45891, NREL 48097, NREL 46526.

NREL prints on paper that contains recycled content. 


\section{Acknowledgments}

ATAMOSTEC acknowledges the strong support of CORFO (the Chilean Economic Development Agency under the contract no. 17PTECES-75830, ATAMOSTEC.

Sandia National Laboratories is a multimission laboratory managed and operated by National Technology \& Engineering Solutions of Sandia, LLC, a wholly owned subsidiary of Honeywell International Inc., for the U.S. Department of Energy's National Nuclear Security Administration under contract DE-NA0003525. 


\section{Executive Summary}

The virtual bifiPV Workshop was held in July 2020 to provide the solar industry with a forum for sharing and discussing research into bifacial photovoltaic (PV) technology. This report outlines major insights from the workshop to give the reader an overview of the latest developments in bifacial PV technology worldwide, from the lab to the field. Citations are drawn from this workshop unless otherwise noted, with all proceedings available online at www.bifipv-workshop.com.

Presentations for the bifiPV2020 Workshop focused on the following areas: bifacial power plant modeling and simulation, albedo improvements, the development of encapsulants, the durability and reliability of current bifacial technologies, performance comparisons between glass-glass and glasstransparent backsheet configurations, the future of passivated emitter and rear contact (PERC) solar cells, and the growing adoption of n-type solar cells.

With $650 \mathrm{GW}$ total PV installed worldwide and 1 TW to come very soon, PERC is now the standard PV cell type produced en masse. However, it is already reaching $23 \%$ efficiency, the upper limit for this type of technology. PV modules breaking the $0.5-\mathrm{kW}$ barrier are starting to appear, and the costs of standard PERC technology are already below 0.2 USD/Wp. In 2019, five GW of bifacial PV were installed worldwide. In 2020, the majority of bifacial installations are expected to be located in the United States, China, and Middle East and North Africa (MENA) states. N-type bifacial technologies are becoming increasingly viable and have huge potential to dominate the market in the coming years. With bifacial technology mounted on horizontal single-axis trackers (HSAT), bids below 10 USD/MWh will soon be observed in the MENA region, and later in Chile and the United States. Factory audits and reliability testing can reduce field failures by helping buyers to select producers that follow rigorous quality assurance and quality control processes. 


\section{Table of Contents}

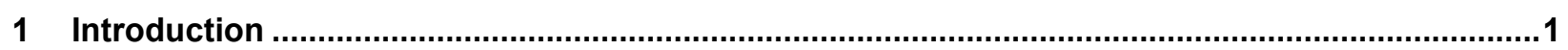

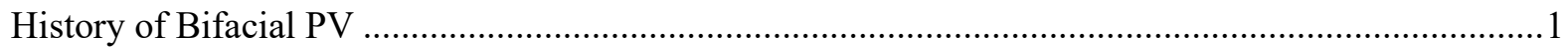

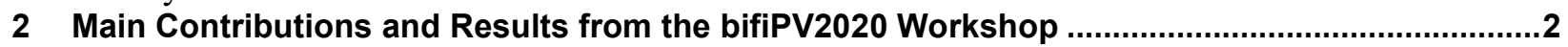

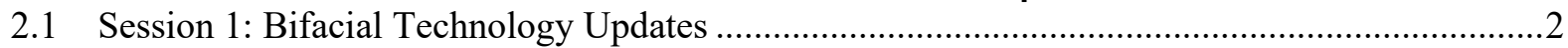

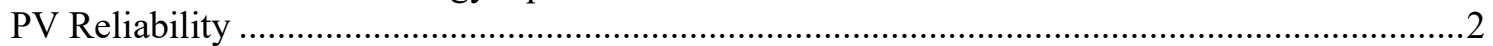

Trends in Bifacial Module Construction....................................................................... 3

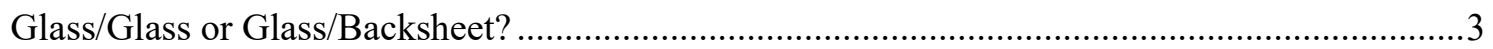

2.2 Bifacial PV Cell Technologies: P-Type and N-Type.....................................................

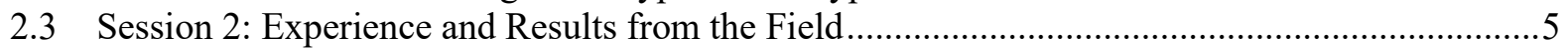

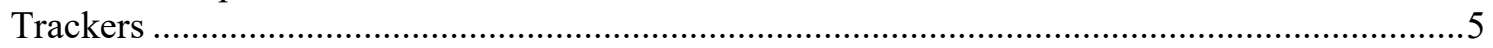

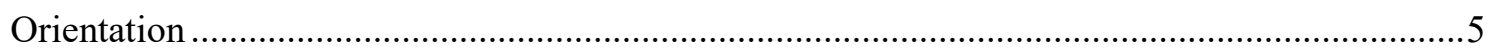

2.4 Session 3 and 4 (Real-World Insights / Reliability and Standards) ........................................6

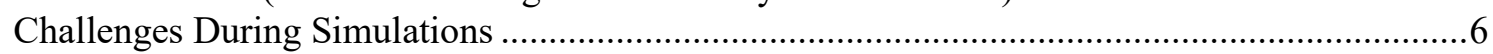

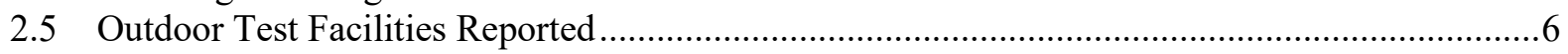

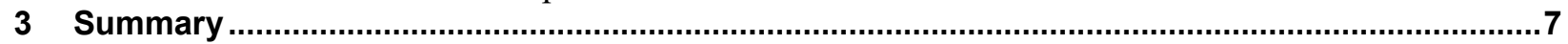

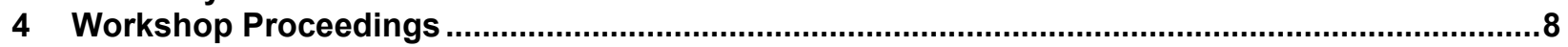




\section{Introduction}

The bifiPV Workshop was established in 2011 as a forum for research on n-type PV technologies. It started in Germany and was supported by ISC-Konstanz, one of ATAMOSTEC's founding partners. The first workshop took place in 2013. At that time, ISC-Konstanz and other research institutions were pushing for n-type and bifacial photovoltaic (PV) technologies to become the market leaders. However, aluminum back-surface field (Al-BSF) polycrystalline technology dominated the market, and very few $\mathrm{R} \& \mathrm{D}$ institutions were conducting applied research on n-type or bifacial PV technologies. Later, as producers transitioned manufacturing lines to passivated emitter and rear contact (PERC) technology, the move to bifacial PV and n-type substrates moved beyond the world of research and became more practical and achievable. ${ }^{1}$

The bifiPV Workshop is a clear example of how research institutions can push the market to adopt innovative products by demonstrating the advancements and bankability of a new technology versus status-quo alternatives. PV modules of $600 \mathrm{~W}$ are starting to appear (at least in a promotional sense), together with new and larger module sizes, but it is important to prioritize increasing the output power of a PV module within the same dimensions (via higher energy density and conversion efficiency) to reduce capital expenditures (CAPEX), increase energy yield, and reduce levelized cost of electricity (LCOE). In 2017, the world's lowest solar PV bid, presented by EDF Energies Nouvelles and leveraging bifacial technology, was well below $20 \mathrm{USD} / \mathrm{MWh}$ at $17.8 \mathrm{USD} / \mathrm{MWh}$ - but it was rejected. The rejection was probably based on the insufficient bankability of bifacial PV. ${ }^{2}$ Since then, many new bifacial PV projects have been developed successfully, strengthening the bankability of the technology. Today, n-type bifacial technologies are becoming more attractive in the market because they can offer performance advantages such as increased bifaciality coefficient, lower temperature coefficient, and reduced susceptibility to initial light-induced degradation (LID). ${ }^{3}$

\section{History of Bifacial PV}

The first solar cell produced in 1954 was an n-type interdigitated back-contact (IBC) solar cell. However, as early solar cells were designed for use in space, $\mathrm{p}$-type silicon PV cells quickly replaced ntype cells because they are more resistant to degradation and the effects of radiation. In the years that followed, Al-BSF cell technology achieved market dominance for terrestrial applications: it offered higher voltages through aluminum $(\mathrm{Al})$ metallization and relatively low costs due to the full $\mathrm{Al}$ coverage. However, Al-BSF cells lack rear-side passivation, so these cells rapidly reached their maximum efficiencies. In response, R\&D efforts began to focus on PERC cells. Many groups started to work in parallel on n-type technologies, including ISC-Konstanz and CEA-INES. By 2016, PERC entered mass production, which was a big breakthrough, and this allowed bifacial technologies to become cheaper because the production process for bifacial is similar to that of PERC. ${ }^{4}$

One of the first challenges for bifacial PV module technologies was the optimization of the rear side: historically, most PV modules have utilized large rear-side junction boxes that block light absorption. Today, half-cut technology and smaller junction boxes have reduced rear-side shadowing. Although there are many advantages of n-type technologies, PERC solar cells still have efficiency-improvement

\footnotetext{
${ }^{1}$ R. Kopecek (ISC-Konstanz), "Bifacial Photovoltaics: Status, Chances and Challenges," bifiPV2020

${ }^{2}$ see:https://www.pv-tech.org/editors-blog/bifacial-technology-was-likely-reason-worlds-lowest-ever-solar-bid-was-reje

${ }^{3}$ R Giehl (Jinko Solar), "Innovation in Bifacial P and N-type Modules to Boost Efficiency and Power Density," bifiPV2020

${ }^{4}$ Ingrid Romijn (ECN), "Bifacial Solar Cells - A Brief Overview,” bifiPV2017
} 
headroom due to the new boost of energy based on Gallium-doped (Ga-doped) silicon wafers, which reduce the effects of LID. ${ }^{5}$

The bifaciality factor of modules (the efficiency of the rear side of the module as compared to the front ${ }^{6}$ ) have improved rapidly, ranging from 0.6 for $\mathrm{p}$-type bifacial PERC to 0.9 and above for $\mathrm{n}$-type - both ntype passivated emitter rear totally diffused (PERT) and heterojunction (HJT) technologies. This improvement in bifaciality factors is increasing the energy yield of n-type bifacial PV technologies, and it also emphasizes the differences between p-type and n-type cells.

Finally, many different project applications are possible with bifacial technology, including vertically mounted building-integrated PV, rooftop projects, and carports. The combination of bifacial PV with horizontal single-axis tracking (HSAT) has been reported as one of the most important next steps in utility-scale PV plants. ${ }^{7}$

\section{Main Contributions and Results from the bifiPV2020 Workshop}

\subsection{Session 1: Bifacial Technology Updates}

The first two workshop speakers addressed emerging trends in bifacial technology, including improvements in module reliability testing and due diligence, and updates to cell and module construction techniques.

\section{PV Reliability}

A primary challenge for PV manufacturers is increasing the reliability and durability of PV modules beyond the standard 25-year warranty while adapting the production process for strong long-term performance in a wider range of climates (temperate, desert, tropical, polar). The materials and architectures used to produce PV modules, as well as characterization and evaluation methods, must be improved and tailored for extreme climates such as desert conditions. The development of ultraaccelerated aging test protocols in the laboratory is therefore central to guaranteeing PV module reliability over time.

Long-term degradation continues to interest the solar community due to the lack of real-world field data available to quantify and characterize bifacial PV degradation rates. PV Evolution Labs (PVEL) identifies potential failure behaviors in the field and replicates them in the indoor lab to improve the industry's understanding of long-term product performance and reliability. PVEL reports that in bifacial modules, front and rear-side degradation can occur at different rates. This could be an issue for energy yield in the field because manufacturers do not provide performance guarantees for bifaciality factors.

Specifically, PVEL's test results show that some encapsulants increase their transparency after thermal cycling, actually improving the performance of the bifacial PV modules, but in general, the front and rear-side degradation are aligned after thermal cycling. However, after ultraviolet (UV) exposure

\footnotetext{
${ }^{5}$ see: https://www.pv-magazine.com/2019/06/15/the-weekend-read-is-perc-still-bankable/

${ }^{6}$ M. Pravettoni (SERIS), "Testing the Tests: Conclusions of the International Bifacial Round-Robin," bifiPV2020

${ }^{7}$ E. Urrejola (ATAMOSTEC) "ATAMOSTEC: Bifacial PV Development for Low LCOE and Field Experience," bifiPV2020
} 
(UV90), the rear side can degrade more significantly than the front side, and the bifaciality factor can be affected by up to $8 \%$. In terms of potential-induced degradation, some PV modules exhibited up to $30 \%$ degradation on the backside, which also impacts the bifaciality factor. With regard to using back-side glass or transparent backsheets, there is insufficient insight into the durability of those materials for bifacial PV as yet; further lab testing and outdoor exposure testing are required. ${ }^{8}$

\section{Trends in Bifacial Module Construction}

A recent major technology change within the PV industry has been the increase in the size of the silicon wafers used in large-scale manufacturing. In 2010, almost all wafers were $125 \mathrm{~mm}$ x $125 \mathrm{~mm}$, but this wafer size almost completely disappeared from the market by 2014, when "M2" (156 mm x $156 \mathrm{~mm}$ ) became the standard size for p-type monocrystalline wafers. Recently, seven China-based PV manufacturers officially announced the establishment of a new wafer size for standard large-area p-type monocrystalline solar cells, the "M10" (182 $\mathrm{mm}$ x $182 \mathrm{~mm})$, which should reduce manufacturing costs. The seven manufacturers are JinkoSolar, JA Solar, LONGi Group, Canadian Solar, Jiangsu Runyang Yueda Photovoltaic Technology Co, Jiangsu Zhongyu Photovoltaic Technology, and Lu'an Solar Technology Co. ${ }^{9}$ As reported by Exawatt, wafer sizes for bifacial PV cells currently vary from M2 to M12 (210 $\mathrm{mm} \times 210 \mathrm{~mm})$. However, not all manufacturers disclose their wafer or cell dimensions, so this figure is based on publicly available information. ${ }^{10}$

\section{Glass/Glass or Glass/Backsheet?}

It was also reported by Exawatt that framed dual-glass PV modules are dominating the bifacial PV market and that manufacturers are moving away from the production of frameless dual-glass PV modules. Glass thicknesses vary from $2 \mathrm{~mm}$ to $3.2 \mathrm{~mm}$. While dual-glass modules with 2-mm glass are currently the most common design, Exawatt expects that modules with transparent backsheets will become more prevalent in the coming years.

Deciding between dual-glass or glass/backsheet designs depends on the PV application as well as the location and environmental conditions of the project site. During the 2020 bifiPV Workshop, some experts mentioned that dual glass may be better suited to harsh environmental conditions, such as those found in the desert or for floating PV plants, due to their higher resistance to extreme climates. ${ }^{7}$ In contrast, transparent backsheets may be preferable due to their lower weight, which reduces transportation and balance-of-systems costs, and due to their lower operating temperatures. The decision must be made based on specific project conditions. As reported by Jinko, there is a temperature dependence on the level of irradiance for glass/glass versus glass/backsheet PV modules, where glass/glass configurations may heat up faster and cool down faster than the glass/backsheet architecture. Jinko's bifacial modules are reportedly using Dupont's transparent Tedlar backsheets. ${ }^{3,11}$

\footnotetext{
${ }^{8}$ J. Meydbray (PVEL), "Bifacial Bake-off: Comparing Technologies and Manufacturers," bifiPV2020

${ }^{9}$ Mark Osborn (PV-tech) "When will large-area M10 wafers be used in high-performance solar modules?" Article, June 28 2020.https://www.pv-tech.org/editors-blog/when-will-large-area-m10-wafers-be-used-in-high-performance-solar-modules

${ }^{10}$ A. Barrows (Exawatt), "Industry Trends in Bifacial Cell and Module Manufacturing," bifiPV2020

${ }^{11}$ K. R. Choudhury (Dupont) "Performance and Long-term Durability of Transparent Backsheets for Bifacial PV Applications," bifiPV2020
} 


\subsection{Bifacial PV Cell Technologies: P-Type and N-Type}

The workshop also featured technology updates from cell and module manufacturers including Jolywood, Jinko Solar, and LONGi.

The talk from Jinko Solar featured details of their shingled module, called "Tiling Ribbon" technology. Tiling Ribbon, similar to other shingling technologies, results from cutting the standard cell size into smaller pieces (typically in half, in thirds, or less are also being employed by some manufacturers) and overlapping the cells slightly to reduce the intercell spaces. This technology will start to appear more frequently in the coming months. ${ }^{12}$

PERC (technology that is based on p-type structures) still dominates the market. At this stage of production, the efficiencies of both bifacial and monofacial PERC are improving. Some experts say PERC has a limitation on production efficiency, while others argue the best method for improving efficiencies is Gallium doping because it results in low or no LID. Selective emitters and passivating contacts are possible solutions, but they need to be cost-effective.

N-type technology is different from p-type due to the doping of the base "bulk" material. In conventional p-type PV technologies, the dopant is boron, but for n-type technologies, the silicon material is doped with Phosphorous before creating the solar cell. N-type has a more promising energy yield due to its higher bifaciality factor, which results from a better rear-side design: this architecture is more symmetrical than those based on p-type technologies. In addition, the temperature conditions during operation seem to be lower for n-type compared to p-type. As presented in the workshop, the leading n-type technologies in the market are likely to be IBC, HJT, tunnel oxide passivated contacts (TOPCON), and nPERT. A move to $\mathrm{n}$-type technologies is expected in the coming years.

The higher cost of bifacial PV technology may only occur at the module step as cell and wafer costs stay constant. The main costs will be the back-junction box, which reduces rear-side shadowing, use of a transparent rear backsheet or rear glass, and use of polyolefin (POE) instead of ethylene vinyl acetate (EVA) for encapsulant material.

Based on n-type's low degradation, low thermal temperatures, and longer lifetimes as compared to ptype architectures, Jolywood has worked on n-PERT since 2016. Today, they are working on TOPCON and IBC technologies with efficiencies higher than $23.5 \%$. Future product generations will be $\mathrm{n}$-IBC and tandem with efficiencies greater than $26 \%$.

Jolywood reported that TOPCON has many advantages: ${ }^{13}$

- N-type materials have a higher lifetime and no LID compared to p-type materials.

- Lower degradation: a reported $1 \%$ degradation for the first year and $0.4 \%$ annually.

- Open-circuit voltages values higher than $700 \mathrm{mV}$ are reached due to the tunnel oxide passivation.

- Lower temperature coefficients $\left(0.32 \% /{ }^{\circ} \mathrm{C}\right)$ that are suitable for desert conditions (similar to HJT).

- Double-side silver fingers, which allow for $>85 \%$ higher bifaciality.

\footnotetext{
${ }^{12}$ R Giehl (Jinko Solar), "Innovation in Bifacial P and N-type modules to Boost Efficiency and Power Density," bifiPV2020

${ }^{13}$ L. Zhifeng (Jolywood), "The Development of Research and Mass production of 24\% Efficiency N-type TOPCon Solar Cell, bifiPV2020
} 
To further increase the efficiency, manufacturers are starting to return to selective emitter solutions and high-quality silicon. Today, n-PERT is produced en masse in Jolywood with average efficiency reaching $23.85 \%$.

\subsection{Session 2: Experience and Results from the Field}

One of the main reasons that doubts remain regarding the outdoor performance of bifacial PV projects versus monofacial is the lack of field data. The main parameter to compare bifacial PV to monofacial is bifacial gain, which indicates the relative increase in energy production of bifacial PV modules in comparison to monofacial modules. At the bifiPV2020 Workshop, several different institutions reported on bifacial gains. The bifacial gain is affected by several factors: the bifaciality factor of the technologies; the albedo of the ground or surrounding areas; and the designs, heights, and pitches of the mounting structures. Depending on the location, albedo can change from a low value (25\%, vegetation) to a medium ( $40 \%$, desert) or high level $(80 \%$, fresh snow). Bifacial gains for "low albedos" were reported to reach up to $6.6 \%$, while for "high albedos," up to $14.5 \%$ gains were reached in California. ${ }^{14}$ Field test reports were also reported by PVEL, comparing their bifacial gains at standard test conditions $-1000 \mathrm{w} / \mathrm{m} 2,25^{\circ} \mathrm{C}$, air mass 1.5 . Preliminary results from PVEL's outdoor study show that bifacial gains vary dramatically, from $8.28 \%$ to $11.44 \%$ across four different module manufacturers over a white groundcover. ${ }^{15}$

\section{Trackers}

The tracker suppliers for bifacial PV technology that presented research at the workshop were Nextracker with $35 \mathrm{GW}$ worldwide (9-GW bifacial), Array Technologies with $10 \mathrm{GW}$, and Soltec with 10.2 GW (3-GW bifacial). There are still questions related to the optimization of tracker systems to achieve higher bifacial PV gains for large-scale projects and related to the impact of higher albedos, as well as the extra cost of applying them.

Soltec began studying bifacial gain in La Silla, Chile, in 2016, and demonstrated a bifacial gain of 13\%. Soltec also reports that jumping from one PV panel in portrait (1P) at a height of $1.35 \mathrm{~m}$ to two PV panels in portrait (2P) at a height of $2.35 \mathrm{~m}$ can deliver an extra yield of $2.1 \%$, and that the average temperature decreases by $3.5^{\circ} \mathrm{C}$ due to a better cooling process. ${ }^{16}$

In contrast, findings from Nextracker showed that for the same ground cover ratio (GCR), 1P may reach a slightly higher bifacial gain compared to $2 \mathrm{P}$. Further, Array Technologies claims there is more bifacial solar resource available for $1 \mathrm{P}$ tracker configurations than for $2 \mathrm{P}$, because $1 \mathrm{P}$ has a higher aspect ratio. ${ }^{17,18}$

\section{Orientation}

The orientation of the PV panels may also play an important role. In many of these test-bed installations, dummy modules are used at the edges of the structures to reduce the shadowing effect, or the edge effect, which may cause variations in bifacial gains. Array Technologies showed that an average bifacial gain of $8 \%$ is reached in the middle of the string, while up to $13 \%$ bifacial gain is reached near the edges

\footnotetext{
${ }^{14}$ H. Fang (LONGI), "Multi GW Bifacial Module Deployment Globally," bifiPV2020

${ }^{15}$ J. Meydbray (PVEL), "Bifacial Bake-off: Comparing Technologies and Manufacturers," bifiPV2020

${ }^{16}$ M. Jiménez Beltrán (Soltec), "Bifacial Tracker and Simulations on PV Plants," bifiPV2020

${ }^{17}$ M. Cooke (Nextracker), "Optimizing Plant Performance with Smart Solar Trackers \& Bifacial Technology,” bifiPV2020

${ }^{18}$ K. Lee (Array Technologies), "Field Testing Meets Modeling: Validated Data on Bifacial Solar Performance," bifiPV2020
} 
of that string. When using 2P tracker configuration, Array Technologies claimed that having a "gap over the torque tube" is not a logical design choice because less energy yield may be expected $(-0.2 \%)$.

\subsection{Session 3 and 4 (Real-World Insights / Reliability and Standards)}

The final days' sessions included speakers presenting on field test results, along with reports on the validation and accuracy of rear irradiance and bifacial performance models.

\section{Challenges During Simulations}

PVsyst is the industry-standard simulation tool, although it has many challenges and cannot accurately estimate the gain from the rear side of bifacial PV modules without significant customization of input variables. Ray tracing requires more computation time, but it more closely models real-world conditions. This is because practical field effects such as spatial variability and the spectrum of reflected irradiance can contribute over $20 \%$ to rear irradiance model differences. ${ }^{19}$

While simulating bifacial PV plants, one of the most important parameters is the rear-side shading factor, but it is also one of the most difficult to estimate. It was reported by Nextracker that in one year, up to $12 \%$ of the light can be blocked from the back of the structure, thus tracking designs that minimize rear side shading are critical. Advanced ray-tracing optical modeling tools (such as Mobidig, ATAMOSTEC/CEA-INES, bifacial_radiance, and PV Lighthouse) can be crucial in providing appropriate PVsyst input parameters to accurately represent real bifacial PV plants. $^{20}$

\subsection{Outdoor Test Facilities Reported}

PVEL: PVEL operates a bifacial test bed at its outdoor PV-USA field laboratory in Davis, California, where nine manufacturers are participating in a comparative study of monofacial and bifacial performance that aims to inform modelling approaches.

SOLTEC-BITEC: The Bifacial Tracker Evaluation Center (BITEC) is a test center in Livermore, California, where different tracking configurations are tested, as $1 \mathrm{P}$ versus $2 \mathrm{P}$ configurations on different albedos for different manufacturers.

CFV Solar Test Laboratory: Array Technologies partners with CFV to perform outdoor testing of trackers, different GCR (ground coverage ratio), and albedos in New Mexico.

NEXTRACKER Bifacial Testing Center: In their outdoor test facility in Fremont, California, Nextracker uses 600-V strings to analyze mismatch, low DC/AC ratio to avoid clipping losses, and other tests.

ATAMOSTEC: Located in the heart of the Desert of Atacama, Chile, ATAMOSTEC tests their own bifacial PV technologies (developed together with CEA-INES and ISC-KONSTANZ) on fixed, vertical mounting systems and on tracking systems at minimodule, module and system levels (see: http://www.atamostec.cl/en).

National Renewable Energy Laboratory (NREL) test facility: NREL operates a 75-kW test bed in Golden, Colorado, with 5 bifacial technologies on trackers: 20 modules $(7.5 \mathrm{~kW})$ per row; 4 strings for

\footnotetext{
${ }^{19}$ S. Ayala Pelaez (NREL), “Ultimate Bifacial Showdown: 75kW Field Results,” bifiPV2020

${ }^{20}$ K. McIntosh (PVLighthouse) "How the PVSyst Inputs for Bifacial Systems Depend on Conditions," bifiPV2020
} 
bifacial PERC, 1 string for bifacial HJT; and 3 strings for PERC monofacial, and module- and stinglevel monitoring and front/rear plane-of-array irradiance assessment. This is one of the only publicly available datasets for bifacial single axis trackers, including module performance, weather, and sensors data [see: https://datahub.duramat.org/dataset/best-field-data]

LONGi test facilities: Through collaboration with partners and direct R\&D efforts, LONGi has multiple GW of deployed bifacial modules and is operating $336 \mathrm{~kW}$ on single-axis trackers in Kubuchi, Inner Mongolia, to evaluate bifacial energy gain.

\section{Summary}

The main contributions of the bifiPV2020 Workshop were in the following areas: bifacial power plant modeling and simulation, albedo improvements, the development of encapsulants, the durability/reliability of current bifacial technologies, performance comparisons between glass-glass and glass-transparent back sheet configurations, the future of PERC solar cells, and the growing adoption of n-type solar cells.

With respect to the modeling and simulation of bifacial power plants, despite efforts to improve models to better represent energy yield and performance, more information is still needed to inform current models. It is a common claim that although ray-tracing theory is fully developed, measurements taken from large-scale power plants are required to truly understand how adjacent structures, ground cover, and other solar modules affect performance. This is very important for two reasons: rear-side reflection is the main advantage of bifacial over monofacial modules, but installation and resulting self-shading between modules and rows may increase module mismatch and therefore reduce energy yield.

Regarding the improvement of albedo, there are several efforts worldwide to artificially increase the reflection of light to the rear side of modules, and thus increase bifacial gains. However, there is still an open question: is the cost of increasing albedo worth the additional energy yield? In terms of extra yield, the answer appears to be yes, but in terms of cost, there is still no clear answer. This introduces a related question: how much should developers and asset owners invest in albedo improvements? This question is particularly difficult to answer for large-scale projects. Given their large size, it is not enough to measure the albedo in just one location-it must be measured in several spots throughout the site.

Currently, the development of encapsulants is a very hot topic in the PV industry. Encapsulants are used in the PV industry to protect the solar cell against environmental conditions that may accelerate degradation, thereby reducing yield and creating potential safety issues. However, it has been found that encapsulants are highly sensitive to UV radiation, especially in desert regions. Because these regions are the most optimal for the development of PV power plants, several companies, laboratories, and institutes, including ATAMOSTEC, are developing alternatives for coping with this challenge. These alternatives range from the development of new encapsulants to coatings that prevent damage from UV radiation, and even to the development of new modules that do not use encapsulants. These products are still in the R\&D stage because new materials may introduce new factors that reduce cell and module efficiency. Furthermore, certain types of cells may interact with these new encapsulants and accelerate degradation. For higher-efficiency technologies, the selection of encapsulants is not trivial. 
The aforementioned developments, particularly encapsulants and coatings, are also related to the increasing attention paid to antisoiling coatings, particularly in light of the significant impact of soiling on performance in desert regions. Indeed, the remoteness of the locations of solar power plants can make cleaning costly and logistically difficult due to the scarcity of water resources. Again, because these regions provide optimal conditions for solar power plants, several companies, laboratories, and institutes are invested in finding solutions, but, as in the case of the encapsulants, these solutions are still in R\&D.

There is also growing interest in the ideal mechanical properties and design of bifacial PV modules as compared to monofacial. There are two main competing designs: the double-glass configuration (with and without frame) and the glass-transparent backsheet configuration. The former has demonstrated positive mechanical properties and can withstand harsh environmental conditions. However, the doubleglass configuration is heavier than current monofacial technologies, and also requires different mounting structures and procedures that could increase CAPEX costs. Designs utilizing transparent backsheets arose as an alternative because they allow bifacial modules to be installed with the same mounting systems used for monofacial modules. However, issues around accelerated degradation of backsheets have raised concerns about the use of transparent backsheets, particularly for projects in desert regions. The question of which configuration is superior remains open, and experiments are taking place worldwide to find an answer. Benchmarking of technologies in desert areas is needed, and ATAMOSTEC is working hard to answer these questions and incorporate the solutions in future BOMs.

Finally, with the increase in available solar cell designs and the popularity of new n-type solar cells, there are questions about the future of PERC cells. N-type solar cells are getting more popular each year due to their higher efficiencies, better operating conditions, longer lifetimes, and reduced initial degradation, compared to p-type solar cells. However, the massive production of n-type solar cells may require high-investment changes in the manufacturing process, which may not be possible for some producers.

In contrast, there is an apparent stagnation in the improvement of p-type solar cells, but they are still in the market, and the popularity of Ga-doped cells is increasing. They are expected to be in the market in mass production for years to come, mostly dominated by PERC. Consequently, it can be deduced that the future of p-type solar cells, especially PERC solar cells, depends upon the industry's ability to reduce the efficiency gap between $n$-type and $\mathrm{p}$-type solar cells and/or maintaining the nontrivial cost differences in the manufacturing process of both types of solar cells.

\section{A factory audit could reduce field failures by helping investors to select producers that follow rigorous quality assurance and quality control processes. These audits are even more critical for future/novel PV technologies.}

\section{Workshop Proceedings}

For further information, download the virtual bifiPV2020 Workshop proceedings: https://www.bifipvworkshop.com/2020-virtualbifipv-proceedings Presentations and video recordings from the workshop are freely available to the public. 